\title{
NON-INTRUSIVE BATTERY HEALTH MONITORING
}

\author{
Laurent GAJEWSKI $^{(1)}$, Celine CENAC-MORTHE ${ }^{(2)}$, Aurore CARRE ${ }^{(3)}$, Patrice SIMON ${ }^{(4)}$, Pierre-Louis \\ TABERNA $^{(4)}$
}

\author{
${ }^{(1)}$ Airbus Defence and Space, 31 rue des cosmonautes - Z.I. du Palays - 31402 Toulouse - France, Email: \\ laurent.gajewski@airbus.com \\ (2) CNES, 18 avenue Edouard Belin-31400 Toulouse - FRANCE,Email:Celine.Cenac-Morthe@.cnes.fr \\ (3) ESTEC, Keplerlaan 1, PO Box 299, 2200 AG Noordwijk, The Netherlands, Email: Aurore.Carre@esa.int \\ ${ }^{(4)}$ CIRIMAT, Université Paul Sabatier - 118, Route de Narbonne - 31062 Toulouse -France, Email: \\ simon@chimie.ups-tlse.fr,taberna@chimie.ups-tlse.fr
}

\begin{abstract}
The "Non-intrusive battery health monitoring", developed by Airbus Defence and Space (ADS) in cooperation with the CIRIMAT-CNRS laboratory and supported by CNES, aims at providing a diagnosis of the battery ageing in flight, called State of Health (SOH), using only the post-treatment of the battery telemetries. The battery current and voltage telemetries are used by a signal processing tool on ground to characterize and to model the battery at low frequencies which allows monitoring the evolution of its degradation with great accuracy. The frequential behaviour estimation is based on inherent disturbances on the current during the nominal functioning of the battery. For instance, on-board thermal control or equipment consumption generates random disturbances on battery current around an average current. The battery voltage response to these current random disturbances enables to model the low frequency impedance of the battery by a signal processing tool. The re-created impedance is then compared with the evolution model of the low frequencies impedance as a function of the battery ageing to estimate accurately battery degradation. Hence, this method could be applied to satellites which are already in orbit and whose battery telemetries acquisition system fulfils the constraints determined in the study. This innovative method is an improvement of present state-of-the-art and is important to have a more accurate in-flight knowledge of battery ageing which is crucial for mission and operation planning and also for possible satellite mission extension or deorbitation. This method is patented by Airbus Defence and Space and CNES.
\end{abstract}

\section{BATTERY MONITORING CONTEXT}

For a satellite, the battery ensures a critical function: store and provide the electrical energy during the entire lifetime of the spacecraft without any interruption. The battery is an electrochemical system which undergoes degradations along its operational life. Typically, its degradation results from the calendar effect and the cycling effect depending on the type of mission. For Geostationary Earth Orbit (GEO) missions, the baseline is presently 15 years in orbit with 1350 battery cycles. For Low Earth Orbit (LEO) missions, the battery has to comply with 55000 cycles during 10 years. The degradations of a battery are characterized by a loss of capacity linked to an increase of the internal resistance. The optimisation of the battery management can be achieved by monitoring its in-flight behaviour and by quantifying its degradation. The battery monitoring can be declined itself in 3 different ways. First way is to check the coherency between predicted degradations and the real ones. Second way is linked to the battery failure and anomaly detection with eventually mission adaptation for recovery. Then, the last is related to the mission extension and deorbitation decision and is in line with the current issue of space debris law.

\section{STATE OF THE ART}

On-ground, the estimation of the battery $\mathrm{SOH}$ is relatively easy and consists to measure the remaining capacity and the DC internal resistance during a complete discharge from a full State of Charge (SoC). However, this method takes a long time (generally several hours) during satellite integration phase. In addition, the transposition on-board is also impossible as a complete discharge in flight means the loss of the satellite. It is also difficult to measure the internal resistance because such a measure needs clean battery current transition.

For this moment, the in-flight battery ageing assessment consists in correlation of the battery voltage evolution with existing steady state battery model. The model is tuned thanks to a fading coefficient in order to match the battery model voltage with the real voltage related to the real current profile. The obtained fading coefficient corresponds to the ageing of the battery. However, there is still large uncertainty on the battery fading because of inherent uncertainty of these models at end of life. The 
voltage observed in flight corresponds to a small visibility window on the discharge $(10-20 \%$ for LEO satellite) and do not allow to know accurately the voltage fall due to no remaining energy in the battery, as shown on the next figure.

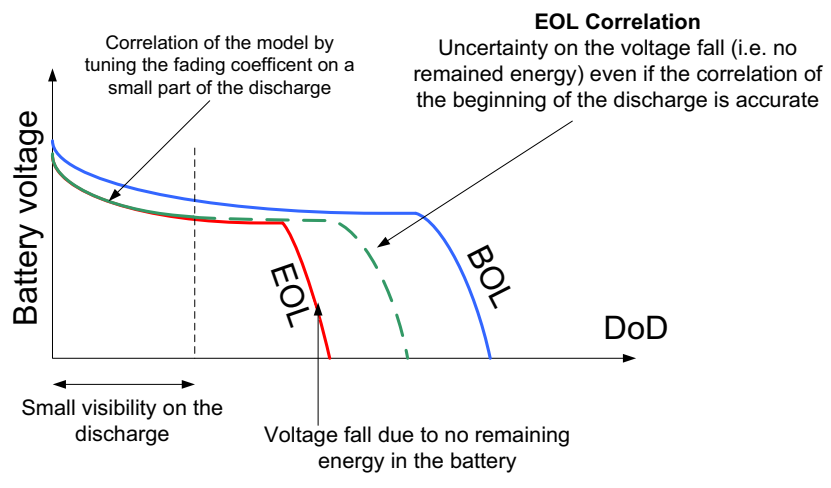

Figure 1. Standard method for battery diagnosis

\section{MONITORING BASED ON IMPEDANCE SPECTROSCOPY}

Previous R\&D studies led by ADS and CIRIMAT (Chemical laboratory in Toulouse) in 2007-2008 pointed out that the analysis of the low frequencies impedance (between $0,1 \mathrm{~Hz}-1 \mathrm{~Hz}$ ) allows characterizing the Li-ion battery ageing. As represented in Fig 2, the impedance of the Li-ion cells increases with the ageing.

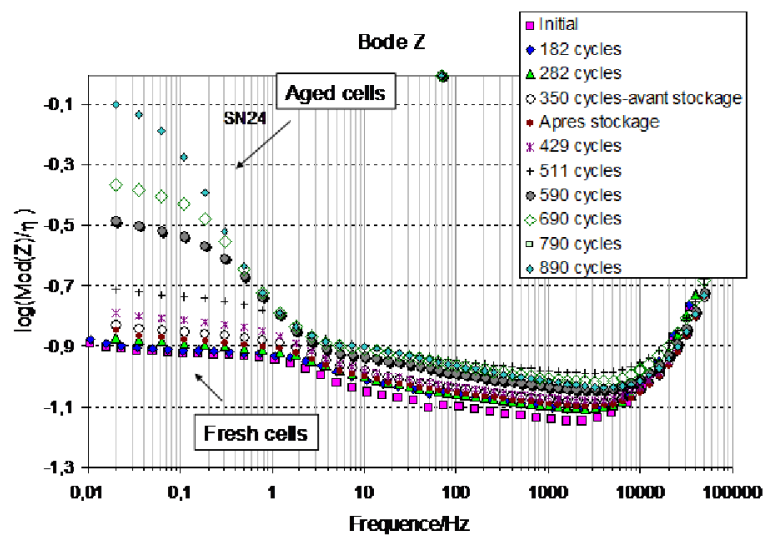

Figure 2. Low frequencies battery impedance evolution due to ageing

A first CNES study has been performed by ADS. Its main purpose was to define a monitoring method of the Low Frequencies (LF) battery impedance, applicable inflight, to determine the ageing. The method, based on impedance spectroscopy, consists in injecting disturbances (low frequencies sinus). As described on Fig. 3, a dedicated module injects the disturbances and acquires the current and voltage of the battery. The recorded TM's are then transmitted to the ground. A post-treatment enables to assess the battery impedance. In parallel, on ground, a reference test has been performed on the same Li-ion accumulator (accumulators, battery string or complete battery) in order to characterise the impedance evolution (impedance measurements) related to the ageing (test of standard capacity and internal resistance). Note that the impedance measurement takes advantage of typical life tests which are nominally performed in frame of satellite programs (to follow the battery performances along the lifetime). The comparison between the measured impedance from in flight data and the impedance measured during the reference test leads to the in-flight battery ageing.

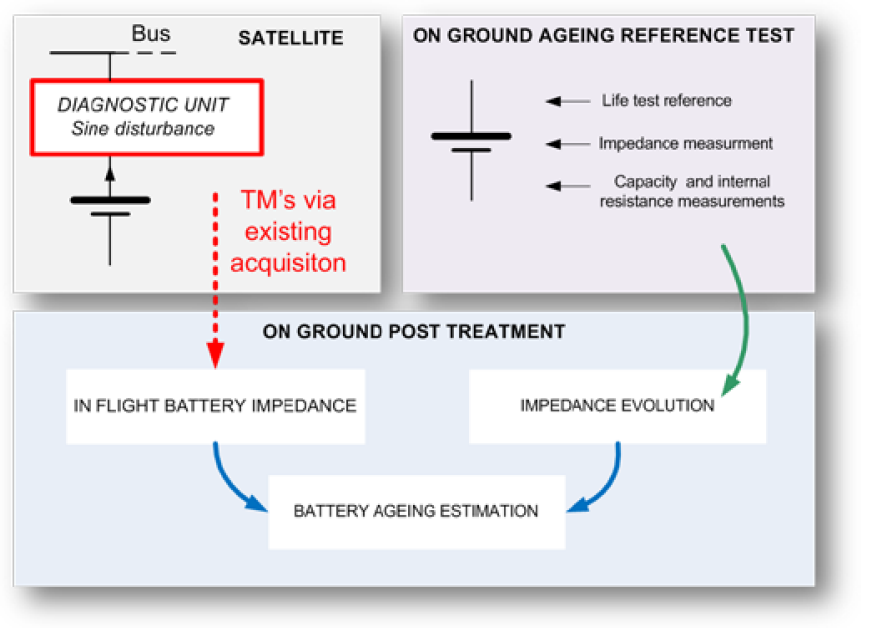

Figure 3. Embedded system for battery monitoring

The method has been proved on a typical LEO cycling life test where an impedance spectroscopy measurement has been regularly performed on $8 \mathrm{~S} 1 \mathrm{P}$ assembly.

The following Nyquist diagram shows the high resolution of ageing and the stability of the measure along the time. Indeed, the evolution of the LF battery impedance as function of the ageing over 10 weeks is visible and corresponds to a really weak ageing (around $1 \%$ of degradation observed on 10 weeks). 


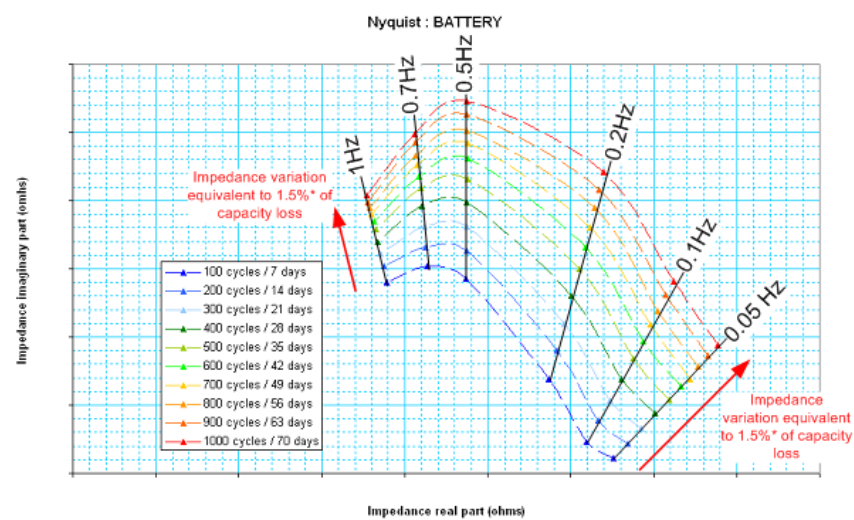

Figure 4. Fine resolution of the ageing thanks to impedance spectroscopy

The battery ageing assessment based on the impedance spectroscopy takes the advantage of the high accuracy of this method. However, for ADS, it is not credible to start the development of a dedicated unit for battery diagnosis which implies cost, time, mass, qualification phase for a non-critical function. Moreover, this system generates disturbances at power bus level for diagnosis which is not convenient at satellite system level.

\section{NON INTRUSIVE BATTERY HEALTH MONITORING PRINCIPLE}

The NiBHM was developed to obtain a diagnosis of the Battery SOH, keeping the accuracy of the impedance spectroscopy method without any on-board unit to perform the diagnosis. It is inspired by the methods of complex systems modelling (bridge, buildings, fans). Indeed, they cannot be easily modelled by physical insights and their characterization from the injection of canonic disturbances (sine, square waves) is generally impossible. The solution consists in using the random natural disturbances applied at the input of the system. First, as shown in Fig 5, the natural disturbances and the system response are measured in time domain. Then, the natural input disturbances are injected into a model, represented by a transfer function. An optimization algorithm enables to select the best model structure (best transfer function form) which is composed by a vector of $n$ optimized parameters. These parameters are optimized so that the difference between the model response and the real system response to real disturbances is minimized.

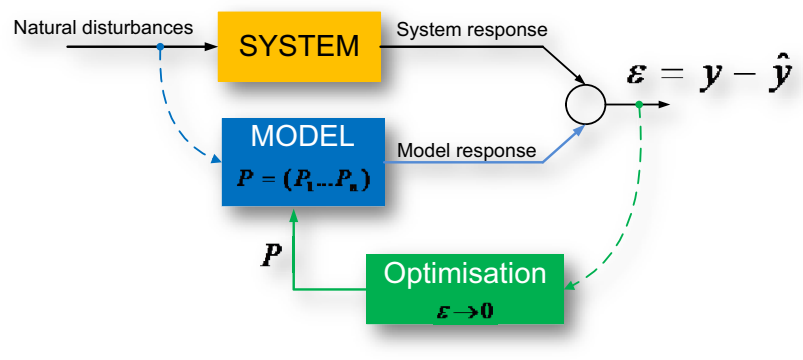

Figure 5. Process of identification based on natural random disturbances

The same principle can be applied to battery. On a spacecraft, the battery current has natural random disturbances, due to the switch on/off of the units and the active thermal control. Hence, from raw battery current and voltage telemetries, the low frequencies impedance can be estimated by post-treatment without any embedded system. Then, if the evolution of the battery impedance as a function of the ageing is known, it is possible to deduce the battery ageing.

The method has been proven thanks to the typical LEO life test previously described. Every 100 LEO cycles (equivalent to one week) an impedance measurement is made between $0.05 \mathrm{~Hz}$ and $1 \mathrm{~Hz}$, thanks to the injection of sine disturbances. Then random disturbances profile is injected on the battery current (this profile is representative of a realistic profile observable on satellite). The battery voltage response is acquired. Finally, the battery impedance is estimated only using the disturbances on the battery current. The method is applied only in discharge so that no additional disturbances due to charge regulation mislead the impedance restitution.

Fig 6 shows the matching between the reference impedance measured on 10 weeks with sine disturbances (in thick lines) and the restitution thanks to the NiBHM process (in large lines). The error of ageing prediction in this demonstration test corresponds to few weeks, which is really accurate (for recall, the ageing on 10 weeks is quantified to $1 \%$ of capacity loss). 


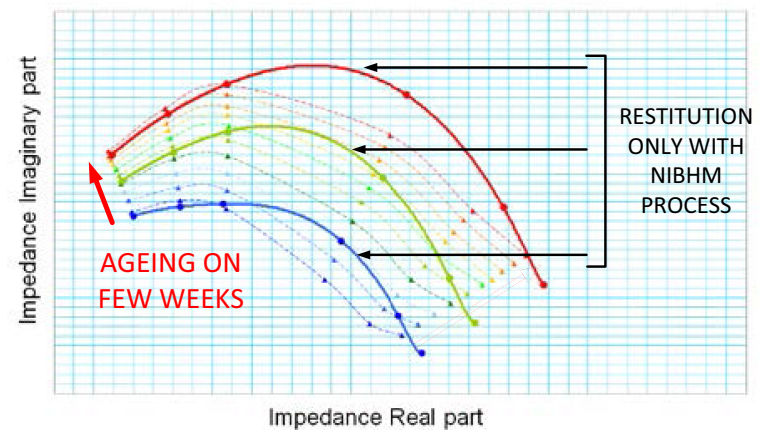

Figure 6. Restitution with NiBHM on a life test

Hence, the restitution of the impedance based on the NiBHM process allows estimating the ageing by a comparison with the set of impedances measurements which are scaled with the capacity loss.

ADS demonstrates also that the TM acquisition systems on present in-flight ADS satellites can be compatible with this method (sampling frequency, Analog-Digital Converter resolution). The real characteristics of several acquisition systems have been emulated on the life test to see the impact on impedance restitution. A dedicated ESA study has been led to quantify the error estimation of the ageing due to uncertainties introduced by acquisition system. It shows also the limitation of oldest ones which are presently in flight.

\section{IN-FLIGHT APPLICATION OF THE NIBHM PROCESS}

The NiBHM process, in flight, is described as follows.

\section{(1) On ground reference test}

The on-ground reference test is necessary to know the LF battery impedance as a function of the ageing. It consists in a life test where regular impedance measurement occurs coupled with capacity and internal resistance measurement.

\section{(2) Raw battery TM's acquisitions}

The raw battery current and voltage telemetries have to be acquired with an optimum sampling rate during a discharge phase on few minutes. The ADC resolution implies some uncertainties which have to be taken into account on the ageing estimation during the post treatment.

\section{(3) Battery ageing estimation}

The post treatment phase is performed on-ground on any computer with NiBHM tool. The tool is able to provide the impedance estimation after filtering, impedance identification and validation. Then, it performs the comparison with evolution law of the impedance as a function of the ageing. The estimation of the ageing is then provided. The post treatment process is really fast: less than 10 seconds to treat a raw data collection for one measure.

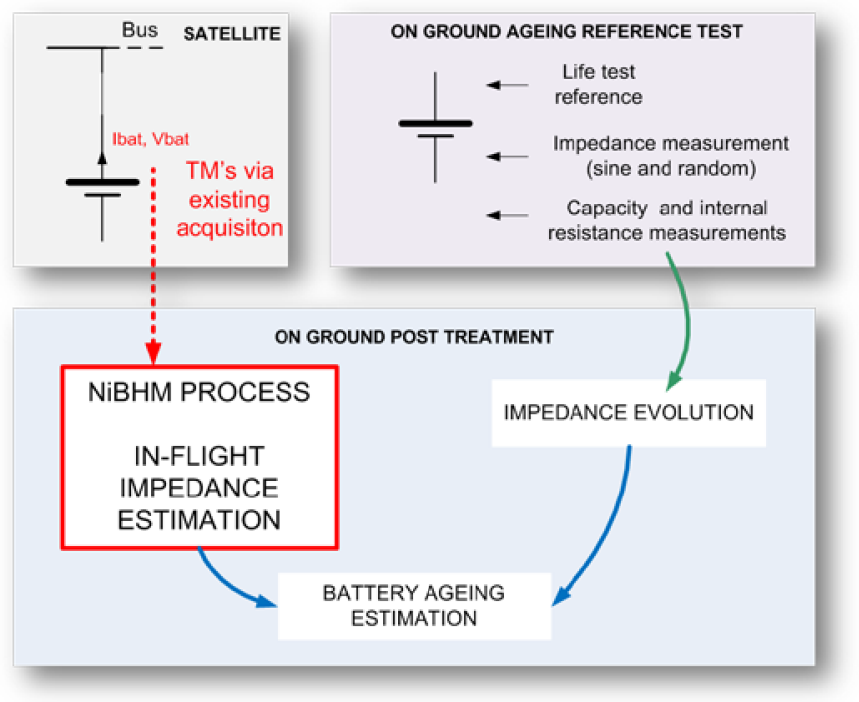

Figure 7. In-flight application of the NiBHM

If no on ground reference test is performed, it is not possible to give an absolute figure of ageing. However, it is possible to monitor the evolution of the impedance which gives a trend for battery degradation. It is also possible to perform the treatment on satellites fleet, to know at the present moment the $\mathrm{SOH}$ of every battery.

\section{IN-FLIGHT APPLICATION ON TRIAL}

The NiBHM process is presently on trial on 2 CNES satellites which have a common technology of battery with the same level of ageing.

Every 6 months, a set of 6 measures (battery current and voltage during several minutes) is performed on each satellite.

Each measure is integrated to NiBHM tool. As no onground reference test exists on this type of battery (because the related life tests were performed 10 years ago), the tool is used for a comparative analysis between the 2 satellites.

Thanks to correlation method (presented in \$2), the observed level of degradation of both batteries is similar. Consequently, the expected result is to provide practically the same battery impedance with NiBHM process. 
As shown, on the next figure, the impedance restitution for each measure is really close from the battery of satellite 1 and the one of satellite 2. The obtained Nyquist diagram allows the restitution of the order of the internal DC resistance of the batteries.

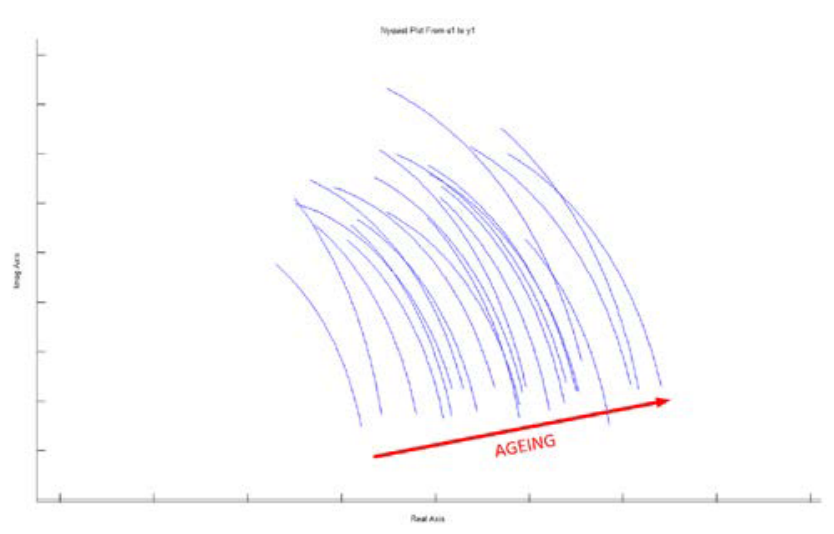

Figure 8. NiBHM on trial on CNES satellitesRestitution of Nyquist diagrams

A present CNES study aims at improving the filtering of raw telemetries in order to reach a better accuracy of the method. The final target for NiBHM process is to estimate the battery ageing with $+/-1 \%$ of accuracy.

\section{CONCLUSION}

In conclusion, based on practically ten years of background, the CNES, ESA and internal ADS studies demonstrates that it is possible to monitor the battery ageing thanks to impedance spectroscopy. The patended NiBHM process developed by ADS from 2011 allows providing a diagnosis based on the accuracy of the impedance spectroscopy, without any modification on present space segment (if the acquisition system onboard fulfils the NiBHM requirements).

On the basis of this promising method, the NiBHM tool can be enriched to enlarge its applicability area by covering a larger range of satellites bus and a larger range of battery temperature for example.

\section{REFERENCES}

1. Gajewski, L., Simon, P. \& Taberna, P.L. (2012) Patent (FR1202266), Procédé d'estimation du viellissement d'une batterie

2. Gajewski, L., (2014), Non-Intrusive Battery Health Monitoring, Space Power Workshop Conference

3. Gajewski, L., Boussouf, L., (2016), Diagnostic batterie non intrusif, CCT CNES conference 